\title{
Cigarette Smoke-Induced Chronic Inflammation Leading to COPD and Lung Cancer: A Multiscale Modeling Study
}

Jie-Lou Liao

Department of Chemical Physics, University of Science and Technology of China, 96 Jinzhai Road, Hefei, Anhui Province, 230026, People's Republic of China

*Corresponding author: Liao JL, Department of Chemical Physics, University of Science and Technology of China, 96 Jinzhai Road, Hefei, Anhui Province, 230026, People's Republic of China, Tel: 86-551-63602323; Fax: 86-551-63602323; E-mail: liaojl@ustc.edu.cn

Received date: February 02, 2017; Accepted date: March 02, 2017; Published date: March 09, 2017

Copyright: (c) 2017 Liao JL. This is an open-access article distributed under the terms of the Creative Commons Attribution License, which permits unrestricted use, distribution, and reproduction in any medium, provided the original author and source are credited.

\section{Mini Review}

An inflammatory response is a general defence mechanism of host immune system to combat invading pathogens [1]. Acute inflammation is a rapid and self-limiting process in which tightly controlled inflammation is critical for host defence, wound healing and maintenance of tissue homeostasis. When the inflammatory cells are not able to eliminate pathogens, however, acute inflammation changes into chronic inflammation that is associated with several disorders including chronic obstructive pulmonary disease (COPD) and lung cancer (LC) [2].

COPD is a chronic inflammatory disease featured by progressive destruction of lung tissues and airway obstruction. Currently, it is the third leading cause of death globally and becomes a major public health burden worldwide [3]. Current drugs are mainly effective in the improvement of symptoms and exacerbations, and there is no cure available for the disease [4]. COPD is caused mainly by cigarette smoking whereas other inhaled pollutants such as indoor cooking and work-place exposures also play an important role in COPD [3]. Although cigarette smoke (CS) is the major risk factor for COPD, only $\sim 20-30 \%$ of long-term smokers develop the disease, demonstrating that disease susceptibility varies significantly among smokers. Cigarette smoking cessation is regarded currently as the most important intervention to prevent COPD progression [5]. But while cigarette smoking cessation can preclude the progression of COPD in some patients (referred as reversibly susceptible smokers), quitting smoking fails to slow or prevent the COPD progression in others, who are referred as severely susceptible smokers [6]. The detailed understanding of different effects of smoking cessation has not been fully obtained.

It is now widely accepted that inflammation and cancer are closely related [7-10]. As the lungs are constantly exposed to environmental insults, which may lead to chronic inflammatory injuries and infection, the link between inflammation and cancer is especially strong in LC patients [11]. Currently, LC is the leading cause of cancer-associated deaths globally with a 5-year survival rate averaging only about $15 \%$ [11]. CS is also the major cause for LC. Despite ongoing efforts for the reduction of smoking prevalence, cigarette smoking still causes $\sim 90 \%$ of lung cancers [12] and $\sim 15 \%$ of lifetime smokers develop the disease [13]. Cigarette smoke (CS) contains more than 8000 components including oxidants such as superoxide and nitrogen oxides and toxins that may cause inflammation, and 73 of which are carcinogens [14]. Long-term exposure of CS to the lung can result in chronic inflammation that generates an inflammatory microenvironment for lung tumor initiation and progression [11].

Since COPD and LC have the same etiological agent, CS, the link between these two diseases has attracted tremendous attention in recent years [15]. Numerous studies have found an increased risk for LC in patients with COPD [12]. LC is about five times more frequently to occur in COPD patients than those without COPD [13]. A most important link between COPD and LC is chronic inflammation $[11,12]$. While COPD is a chronic inflammatory disease, dysregulated inflammation in COPD is critical for increasing risk of LC [11]. However, an issue is raised regarding the profiles of immune cells in COPD and LC patients. As COPD and LC are diametrically opposed in nature, their immune cell profiles would be very different $[2,12]$. In COPD, the immune cells such as CD8+ T cells are cytotoxic (often pro-inflammatory) predominantly, while the immune cells, e.g., myeloid-derived suppressive cells (MDSCs), in LC are often anticytotoxic and immunosuppressive [2,12]. How this apparently contradictory cell profile is achieved in an LC patient with COPD is not fully understood. Although CS exposure experiments in rodent can reliably reproduce emphysema [16], they cannot produce lung cancer because rodents have different anatomy of the upper respiratory tract so that they are obligate nose-only breathers [14]. Despite significant advances in using $\mathrm{A} / \mathrm{J}$ mice to develop adenomas upon CS exposure, it is still very difficult to study both diseases in experiments [12], in particular, to measure the temporal sequence of inflammation in these diseases in an animal model or a human subject as COPD and LC both are complex and progressive chronic disorders. Here, computer modelling can be of great help to study the dynamic properties of COPD and LC upon CS exposure and the mechanistic links between these two diseases.

A CS-induced chronic inflammatory response involves both innate and adaptive immune system and is mediated by a complicated network consisting of multiple immune cell types, molecular mediators, and tissues. This network bears a feature with multiple temporal and spatial scales. For example, cytokine regulation of signal transduction for cell function usually happens on a sub-second timescale, while cell secretion of cytokines takes minutes to hours [17].

To describe CS-induced COPD progression, we proposed a multiscale network model as shown in Figure 1. In this network model, the nodes represent important cytokines, immune cells, and lung tissues, whereas the edges represent the interactions between these network components [17]. The network dynamics for the cytokines, immune cells and tissue damage (TD) can be evaluated by a set of ordinary differential equations (ODEs) [17]. In our modelling study, several positive feedback loops and network components are identified, playing a determinant role in the CS-induced inflammatory response in COPD progression. The results in this study have shown that CSinduced COPD progression is a multistep process from an acute to a chronic phase. In the acute phase, the innate immune response predominates. During the transition from an innate to an adaptive response, if M1 (denoted as pro-inflammatory macrophage) cells 
predominate over M2 (denoted as anti-inflammatory/regulated macrophage) cells, the system will proceed to high-grade chronic inflammation and eventually toward stable COPD, in which the adaptive immunity plays a predominant role. But when M2 (Treg) cells are dominant over M1 (Th17 and CD8+ T) cells, the acute inflammation turns into the low-level chronic inflammation, and COPD does not happen. Our simulations offer rationales for the above-mentioned issues regarding CS-induced COPD as seen in Figure $2 \mathrm{a}$ and $2 \mathrm{~b}$ [17]. Different smokers have distinct parameters, to which the TD outcome is sensitive, in the ODEs. For example, different values of the parameter, k13, which represents the rate constant of the M1 contribution to $\mathrm{TD}$, correspond to smokers with different levels of susceptibility. Figure 2 shows that when $\mathrm{k} 13$ is less than $2.6 \times 10^{-2} \mathrm{ml} /$ (cell day), TD remains at relatively low level $(<30 \%)$, showing a COPD resistant feature. While k13 lies in a value range between $2.6 \times 10^{-2}$ and $0.31 \mathrm{ml} /$ (cell day), COPD can happen, however, smoking cessation results in TD decreasing to the baseline (Figure $2 b$ ). In this situation, COPD is reversible. When $\mathrm{k} 13$ is larger than $0.31 \mathrm{ml} /$ (cell day), TD is reduced to some extent at the steady state, but still remains at a high level (larger than 30\%) after smoking cessation. Therefore, our simulations disclose that there are three types of smokers in accordance with their COPD susceptibilities, i.e., resistant, reversely susceptible and severely susceptible smokers. While long-term cigarette smoking can cause just low-grade chronic inflammation in resistant smokers but without COPD, the disease can occur in susceptible smokers under the same CS exposure conditions (Figure 2). After CS cessation, COPD can be stopped in reversibly susceptible smokers, whereas the disease cannot be completely reversed in severely susceptible smokers, as shown in (Figure 2).

To study CS-induced LC initiation and progression, the above COPD-associated network model was extended by adding lung tumor (LT) and associated cells and molecular mediators [18]. In this model, several LT-related positive feedback loops are identified. For instance, while $\mathrm{CD} 8+\mathrm{T}$ cells performs antitumor function, LTs can secret checkpoint molecules such as the programmed death-1 ligand (PD-L1) or cytotoxic T lymphocyte antigen-4 (CTLA-4) to down-regulate the antitumor activity of CD8+T cells [19]. Therefore, the positive feedback loops, LT $\rightarrow$ PD-L1 (CTLA-4) CD8+T LT, are formed, playing a critical role in LC progression. Targeting these immune checkpoint molecules to unleash a patient's own T cells is revolutionizing cancer therapies [20]. In addition, LT produces macrophage colonystimulating factor (M-CSF) to recruit MDSCs that suppress the antitumor activities of $\mathrm{CD} 8+\mathrm{T}$ cells, forming another positive feedback loop, LT $\rightarrow$ M-CSF $\dashv$ CD8+T $\dashv$ LT. MDSCs also secret IL-6 and TGF- $\beta$ to activate Th-17 that produces IL-17 to promote the recruitment of MDSC, i.e., MDSC $\rightarrow$ (IL-6+TGF- $\beta$ ) $\rightarrow$ Th17 $\rightarrow$ IL17 $\rightarrow$ MDSC [21]. While IL-17 can promote antitumor activity through the activation of CD8+T cells, MDSC thus plays both pro-tumor and antitumor roles in LC development. Indeed, immune cells often perform such dual functions in tumor initiation and progression. Our modeling study has demonstrated that CS-induced LC development is a multiphase process and chronic inflammation in COPD progression generates a microenvironment for LT initiation and progression [18].

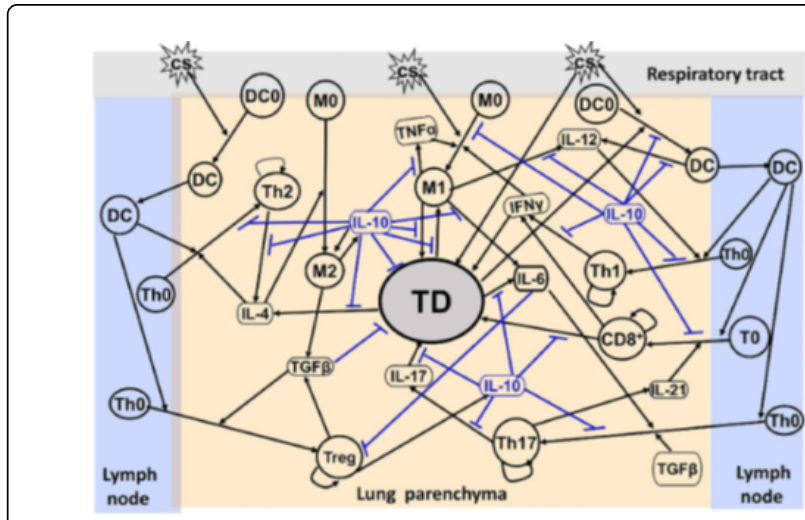

Figure 1. Network model for CS-induced immune response. There are two types of inputs initiated from a node: a positive or an upregulation input (denoted by $\rightarrow$ ) indicates that an increasing of the concentration of the tail node will lead to an increasing of that of the head node or an up-regulation of the process when the input arrow ends at an edge between two nodes, and vice versa for a negative or a down-regulation (inhibition) input (denoted by $\dashv$ ). Interactions between various nodes that represent cytokines, immune cells, and TD are described [17].

CS: Cigarette Smoke; M0: Immature Macrophage; M1: Classically Activated Macrophage; M2: Alternatively Activated Macrophage; DC0: Immature Dendritic Cell; DC: Activated Dendritic Cell; Th0: Immature T helper 1 Cell; Th1: T helper 1 Cell; Th2: T helper 2 Cell; Th17: T helper 17 Cell; T0: Immature Cytotoxic T Cell; CD8+: CD8+ T Cell; Treg: Regulatory T Cell; TNF- $\alpha$ : Tumor Necrosis Factor- $\alpha$; IFN- $\gamma$ : Interferon- $\gamma$; TGF- $\beta$ : Transforming Growth Factor- $\beta$; TD: Tissue Damage.

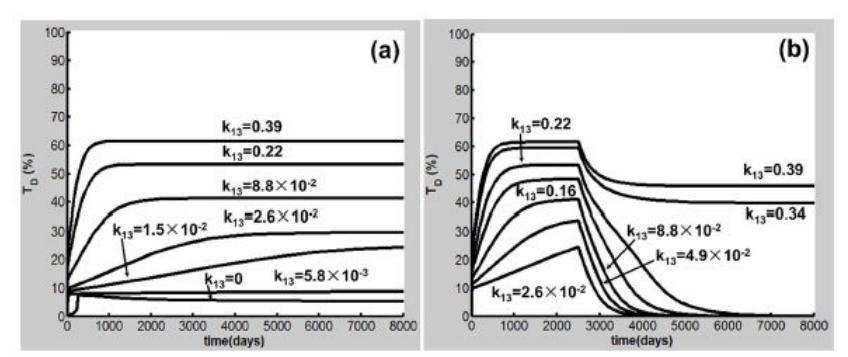

Figure 2. Dynamics of TD with different values of $\mathrm{k} 13$ and effects of cigarette smoking cessation. (a) $\mathrm{k} 13<2.6 \times 10^{-2} \mathrm{ml} /$ (cell day) corresponds to resistant smokers ( $\mathrm{TD}<30 \%$ ), while k13 $\geq 2.6 \times 10^{-2}$ $\mathrm{ml} /$ (cell day) is associated with susceptible smokers. (b) Effects of smoking cessation after 2500 days of CS exposure. $2.6 \times 10^{-2} \mathrm{ml} /$ (cell day) $\leq \mathrm{k} 13<0.31 \mathrm{ml} /$ (cell day) corresponds to reversible susceptible smokers and COPD is reversible. $\mathrm{k} 13 \geq 0.31 \mathrm{ml} /$ (cell day) is associated with severely susceptible smokers. In this case, COPD is not reversible.

CS-induced chronic inflammation resulting in COPD and LC is a highly complicated and dynamic process, which involves tremendous amounts of interactions between molecular mediators, immune cells and lung tissues. Multiscale modeling offers a useful approach to 
Citation: Liao JL (2017) Cigarette Smoke-Induced Chronic Inflammation Leading to COPD and Lung Cancer: A Multiscale Modeling Study. J Immuno Biol 2: 119. doi:10.4172/2476-1966.1000119

Page 3 of 3

elucidate the cellular and molecular mechanisms of these diseases and to explore the mechanistic links between them.

\section{Acknowledgment}

The author's work presented in this review has been supported by the National Natural Science Foundation of China under Grants 21273209 .

\section{References}

1. Balkwill F, Charles KA, Mantovani A (2005) Smoldering and polarized inflammation in the initiation and promotion of malignant disease. Cancer Cell 7: 211-217.

2. Houghton AM (2013) Mechanistic links between COPD and lung cancer. Nat Rev Cancer 13: 233-245.

3. Rennard SI, Drummond MB (2015) Early chronic obstructive pulmonary disease: definition, assessment, and prevention. Lancet 385: 1778-1788.

4. Barnes PJ (2013) New anti-inflammatory targets for chronic obstructive pulmonary disease. Nat Rev Drug Discov 12: 543-559.

5. Barjaktarevic IZ, Arredondo AF, Cooper CB (2015) Positioning new pharmacotherapies for COPD. Int J Chron Obstruct Pulmon Dis 10: 1427-1442.

6. Faner R, Cruz T, Agusti A (2013) Immune response in chronic obstructive pulmonary disease. Expert Rev Clin Immunol 9: 821-833.

7. Hanahan D, Weinberg RA (2011) Hallmarks of cancer: the next generation. Cell 144: 646-674.

8. Coussens LM, Zitvogel L, Palucka AK (2013) Neutralizing tumor promoting chronic inflammation: a magic bullet? Science 339: 286-291.

9. Coffelt SB, De Visser KE (2014) Cancer: Inflammation lights the way to metastasis. Nature 507: 48-49.

10. Crusz SM, Balkwill FR (2015) Inflammation and cancer: advances and new agents. Nat Rev Clin Oncol 12: 584-596.
11. Bozinovski S, Vlahos R, Anthony D, McQualter J, Anderson G, et al. (2016) COPD and squamous cell lung cancer: aberrant inflammation and immunity is the common link. British J Pharmacol 173: 635-648.

12. Houghton AM, Shapiro SD (2015) Inflammation and lung cancer: The relationship to chronic obstructive pulmonary disease. Infl lun cancer. $1-22$.

13. Rigden HM, Alias A, Havelock T, O'Donnell R, Djukanovic R, et al. (2016) Squamous metaplasia is increased in the B ronchial epithelium of Smokers with chronic obstructive pulmonary disease. PLoS One 11: e0156009.

14. Flora SD, Ganchev G, Iltcheva M, Maestra SL, Micale RT, et al. (2016) Pharmacological modulation of lung carcinogenesis in smokers: Preclinical and clinical evidence. Trends Pharmacol Sci 37: 120-142.

15. Gonzalez J, Marín M, Sánchez-Salcedo P, Zulueta JJ (2016) Lung cancer screening in patients with chronic obstructive pulmonary disease. Ann Transl Med 4: 160

16. Hautamaki RD, Kobayashi DK, Senior RM, Shapiro SD (1997) Requirement for macrophage elastase for cigarette smoke-induced emphysema in mice. Science 277: 2002-2004.

17. Pan Z, Yu H, Liao JL (2016) Probing cellular and molecular mechanisms of cigarette smoke-induced immune response in the progression of chronic obstructive pulmonary disease using multiscale network modeling. PLoS One 11: e0163192.

18. Pan Z, Yu H, Liao JL (2017) Cellular and molecular mechanisms of inflammation linking lung cancer with COPD revealed by network modeling.

19. Coussens LM, Zitvogel L, Palucka AK (2013) Neutralizing tumorpromoting chronic inflammation: a magic bullet? Science 339: 286-291.

20. Mahoney KM, Rennert PD, Freeman GJ (2015) Combination cancer immunotherapy and new immunomodulatory targets. Nat. Rev Drug Discov. 14: 561-584.

21. Kitamura T, Qian BZ, Pollard JW (2015) Immune cell promotion of metastasis. Nat Rev Immunol. 15: 73-86. 